\title{
Severe congenital hypochromic anemia with ringed sideroblasts
}

INSERM

\section{Source}

INSERM. (1999). Orphanet: an online rare disease and orphan drug data base. Severe congenital hypochromic anemia with ringed sideroblasts. ORPHA:300298

STEAP3/T SAP6-related sideroblastic anemia is a very rare severe non-syndromic hypochromic anemia, which is characterized by transfusion-dependent hypochromic, poorly regenerative anemia, iron overload, resembling non-syndromic sideroblastic anemia (see this term) except for increased erythrocyte protoporphyrin levels. 